\title{
A Broadband Multi-layer Dual-polarization Slot Antenna Based on Differential-feed
}

\author{
Jin-dong $\mathrm{LI}^{1}, \mathrm{Ge} \mathrm{GAO}^{2}$, Run-dong $\mathrm{ZHENG}^{3}$ and Ji-bin $\mathrm{LIU}^{4}$ \\ ${ }^{1}$ Navy navigation support factory, Qingdao, Shandong province, China \\ ${ }^{2,3,4}$ College of Electronics Science and Engineering, National University of Defense \\ Technology, Changsha, Hunan province, China
}

Keywords: Slot antenna, Wideband, Dual-polarization, Differential-feed.

\begin{abstract}
In this paper, a broadband dual-polarization slot antenna based on differential-feed at $\mathrm{X}$ band is proposed. By adopting wide round slots, the antenna inherits a good advantage of broad band. And the multi-layer structure achieves a good isolation between the two ports. In order to improve the high cross polarization level (XPL) and pattern distortion for wide slot antenna, differential feed is introduced. Simulation results shows that the proposed antenna obtains a $27 \%$ and $30 \%$ return loss bandwidth below $-10 \mathrm{~dB}$ at each port respectively. The port isolation is below $-20 \mathrm{~dB}$ within the common band. The largest XPL in the range of half-power angle is $-15 \mathrm{~dB}$. And the gain is $7.5 \mathrm{dBi}$ to $8.5 \mathrm{dBi}$ for port 1 and $6.5 \mathrm{dBi}$ to $9.5 \mathrm{dBi}$ for port2.
\end{abstract}

\section{Introduction}

The planar slot antenna has the characteristics of small volume, low profile, low cost and simple integration. It has good paperback structure and is suitable for low-profile and embedded installation[1]. Due to these good features, planar slot antenna is widely used in broadcasting, satellite communications, airborne or missile-borne radar and meteorological, navigation, beacon, etc. With the rapid development of conformal technology, the planar slot antenna will occupy a more important position.

In 1989, J. Hirokawa proposed the idea of using wide slots to extend the band width [2]. The rectangular wide-slot slot antenna in [2] achieves a return loss bandwidth of 35\%. When using circular slot or elliptical slot, the wideband effect of the rectangular wide slot antenna can also be realized. In [3,4], a rounded microstrip line feeds a circular slot antenna, and through adjusting their relative position can achieve an impedance bandwidth of $82 \%$ and $127 \%$ respectively. [5] deforms a circular slot to a P-shaped slot, and feeds by a simple $50 \Omega$ transmission line. The antenna can work stably in the range of a bandwidth of $70 \%$.

In terms of dual-polarization slot antennas, [6] uses an annular slot and two mutually perpendicular microstrip lines to achieve dual polarization. By adding a conductor strip line on the opposite side of the feed line, the isolation between the two ports lowers to $-33 \mathrm{~dB}$ and the cross-polarization level reaches below -30dB. But the bandwidth is only $4 \%$ at $2.4 \mathrm{GHz}$. [7] adopts a Y-shaped wide slot and improves the isolation by extending a length at the center. The antenna achieves a bandwidth of $14.2 \%$ with an isolation of less than $-15 \mathrm{~dB}$. The antenna proposed in [8] is two orthogonal dipole slots with an isolation of less than $-35 \mathrm{~dB}$ and a cross-polarization level of less than $-27 \mathrm{~dB}$. By introducing the stepped-impedance structure, the antenna bandwidth reaches $38.7 \%$.

In order to realize a broadband co-planar dual-polarization antenna, this paper proposed a multi-layer slot antenna based on differential feed. Adopting wide round slots keeps the good 
characteristics of broad band and port symmetry. And multi-layer structure increases the isolation between the two polarization. By introducing differential feed, the bad XPL of wide slot antenna is improved, leading to a higher polarization purity and a more stable pattern within the working band.

\section{Antenna Configuration}

Fig. 1 shows the configuration of the proposed dual-polarization slot antenna. It consists of triple-layer metal slots, four anti-phase discrete ports of two polarization, four layers of substrate, an air gap and a planar reflector. The multi-layer configuration increases the isolation between the two polarization, and also forms a strip-line feed structure, which has less stray radiation and impedance characteristic insensitive to frequency, thus sustaining broader band and better patterns. To suppress back radiation, a planar metal reflector is introduced below an air gap with a height of $h c$. The proposed slot antenna has overall dimensions of $W \times W$. The substrate adopts Rogers RT5880 with permittivity 2.2, with each layer height is $h s$. Three round slots of diameter $R s$ are etched on the triple metal layer. One polarization is realized by two same anti-phase discrete ports on the same layer in order for higher polarization purity. The ports of two polarization excites the same radiation slots, which realizes co-planar dual-polarization and higher aperture efficiency. The key structure parameters are optimized and the final values are given in Fig.1.
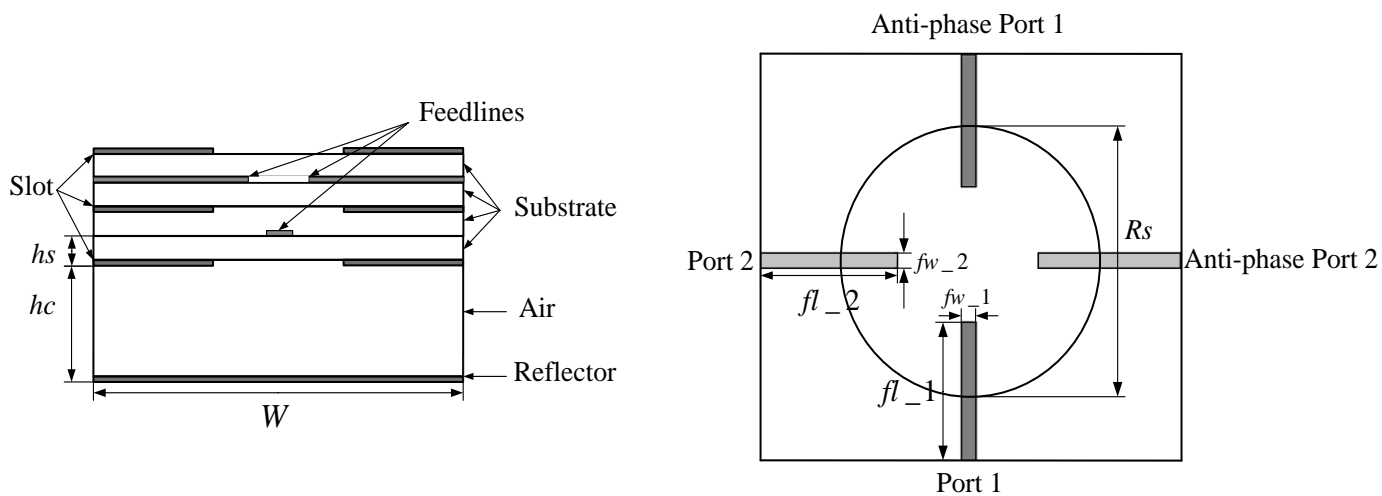

Figure.1 Geometry of the proposed wideband dual-polarization slot antenna. The key parameters are fixed as $\mathrm{W}=25 \mathrm{~mm}, \mathrm{Rs}=20 \mathrm{~mm}, \mathrm{hs}=1 \mathrm{~mm}, \mathrm{hc}=4 \mathrm{~mm}, \mathrm{fl} \_1=9 \mathrm{~mm}, \mathrm{fw} \_1=\mathrm{fw} \_2=0.5 \mathrm{~mm}, \mathrm{fl} \_2=8 \mathrm{~mm}$.

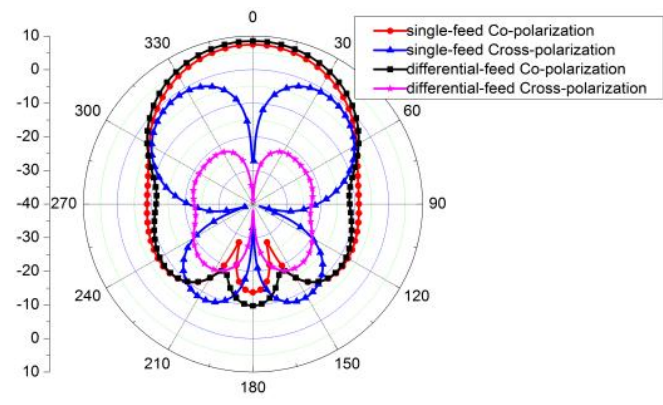

Figure. 2 Cross polarization at $\mathrm{H}$ plane at $10.65 \mathrm{GHz}$ of single-feed and differential-feed

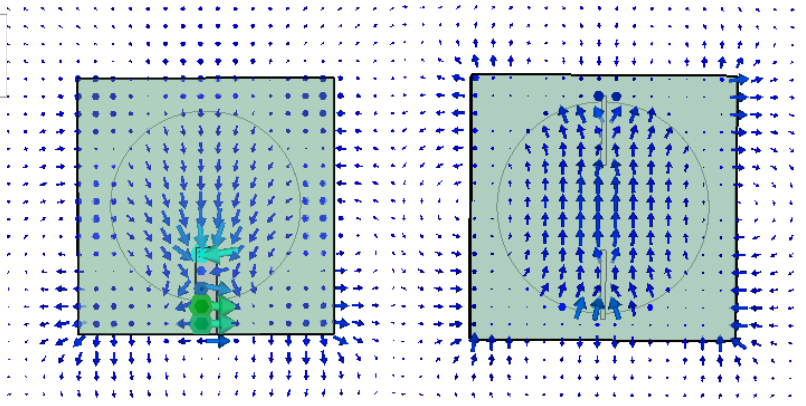

(a)single-feed (b)differential-feed

Figure. 3 Aperture field distribution at $10.65 \mathrm{GHz}$

For slot antenna, the imbalance of single-feed will occur when slot gets wider, which will have a bad influence on radiation characteristics. Beam at E plane will squint, and patterns at $\mathrm{H}$ plane will split due to high cross polarization. Therefore, dual-port balanced feed is introduced to improve the problem. To demonstrate the effect of differential-feed on antenna radiation, 
aperture field distribution and $\mathrm{H}$ plane cross polarization at $10.65 \mathrm{GHz}$ are given in Fig.2 and Fig.3. As it can be seen in Fig.2, when differential-fed the cross polarization decreases by about $-20 \mathrm{~dB}$. The improvement can be explained from the aperture field distribution showed in Fig.3. After adding an anti-phase port, the field distribution gets more uniform and symmetric, which suppresses the cross polarization.

\section{Parametric Analysis}

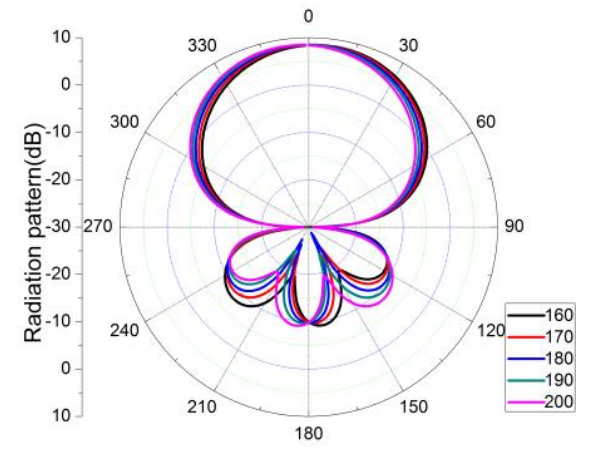

Figure.4 Radiation patterns at E plane at $10.65 \mathrm{GHz}$ under different phase differences

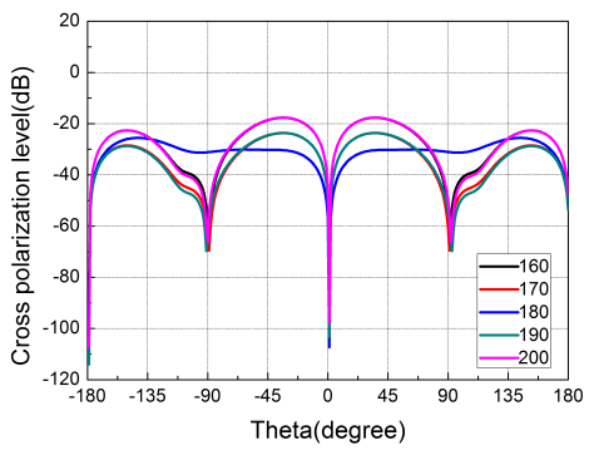

Figure.5 Cross polarization levels at $\mathrm{H}$ plane at $10.65 \mathrm{GHz}$ under different phase differences

Differential-feed requires a $180^{\circ}$ phase difference between the two ports theoretically. But in practical application, it is difficult to realize strict broadband anti-phase. Fig.4 and Fig. 5 shows the radiation patterns at E plane and cross polarization levels at $\mathrm{H}$ plane with phase differences ranging from $160^{\circ}$ to $220^{\circ}$. From Fig. 4 we can see that the antenna gain and pattern remains almost the same when the phase difference changes, except a little beam squint. The more distinct the phase difference is from $180^{\circ}$, the more the maximum radiation direction deviates from the antenna normal. Fig.5 demonstrates that the antenna has a lowest cross polarization level at $180^{\circ}$. And the XPL near the maximum radiation direction will increase about $7 \mathrm{~dB}$ when the phase difference is $10^{\circ}$ bigger or smaller.

Set the port phase difference as $180^{\circ}$ at $10.65 \mathrm{GHz}$ and observe the effects of $R s$ and $h c$ on return loss of two ports when other parameters are fixed. Experiments shows that the resonant frequencies and variation rule of two ports are almost the same with parameter changing. Here the relevant curves of Port 1 are showed in Fig. 6 and Fig.7. We can see that $R s$ has an impact on the impedance match and with $R s$ increasing, the resonant frequencies get higher. The height $h c$ also influences the resonant depth, and when $h c=4$ the return loss curve has two resonant frequencies thus obtaining the broader band.

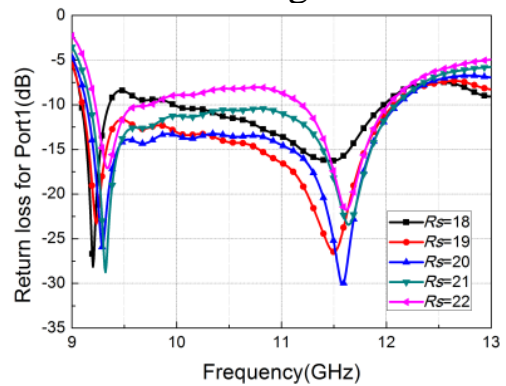

Figure.6 Effects of $R s$ on Port 1 return loss

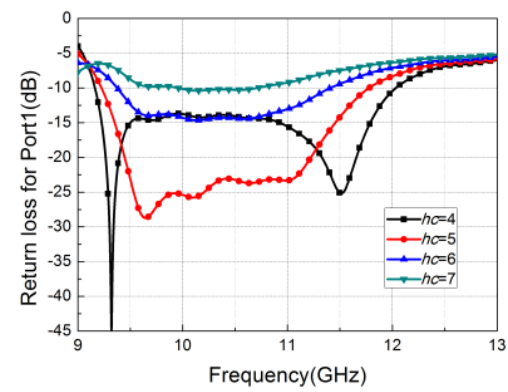

Figure.7 Effects of $h c$ on Port 1 return loss 


\section{Simulation Results}

Simulation results of the proposed dual-polarization slot antenna are given below. As shown in Fig.8, the return loss of Port 1 is below $-10 \mathrm{~dB}$ from $9.15 \mathrm{GHz}$ to $12 \mathrm{GHz}$, and the return loss of Port 2 is below $-10 \mathrm{~dB}$ from $9.3 \mathrm{GHz}$ to $12.6 \mathrm{GHz}$. The isolation between the two ports is below $-20 \mathrm{~dB}$ within the common band. The gain for each port is given in Fig.9. We can see that the gain for port 1 is more stable than port 2 within the working band. And the radiation patterns are showed in Fig.10. The patterns of two polarizations have a good consistency, and the XPL is below $-15 \mathrm{~dB}$ within the band from $9.3 \mathrm{GHz}$ to $12 \mathrm{GHz}$.

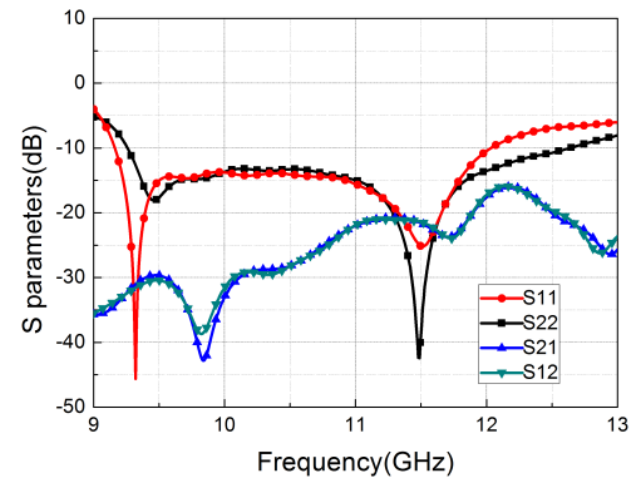

Figure.8 S parameters of the proposed antenna

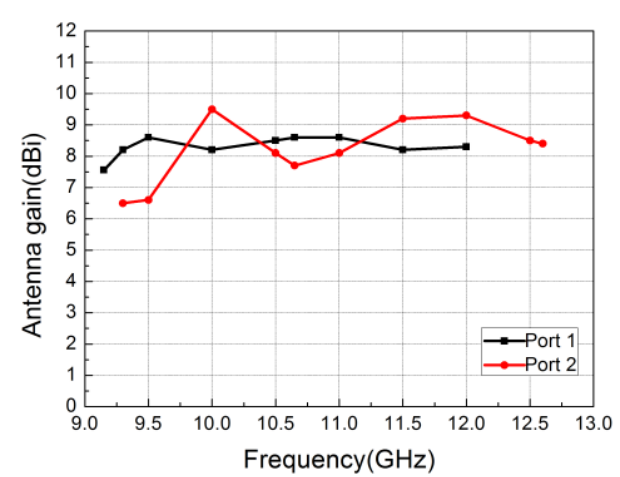

Figure.9 Gain of the proposed antenna

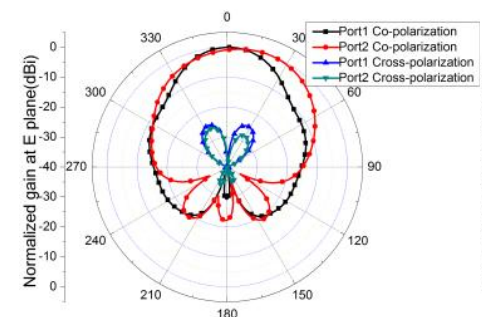

(a) $9.3 \mathrm{GHz}$ at E plane

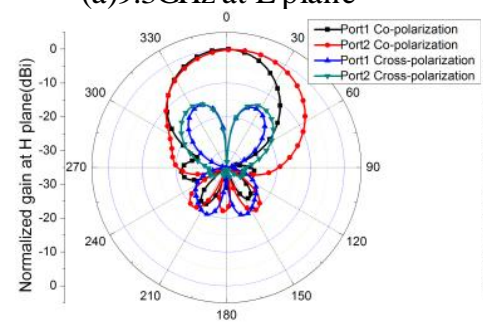

(d)9.3GHz at H plane

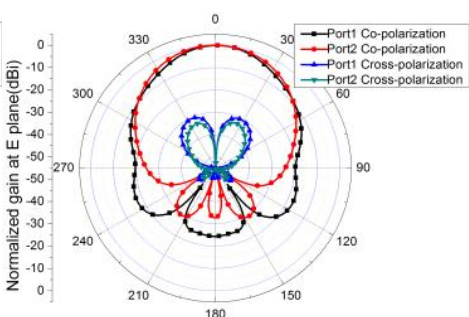

(b) $10.65 \mathrm{GHz}$ at E plane

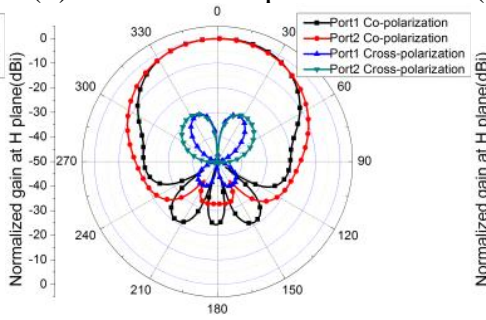

(e)10.65GHz at $\mathrm{H}$ plane

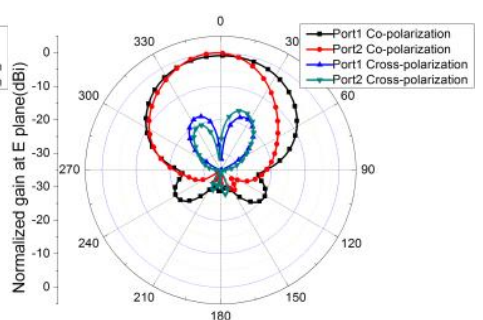

(a) $12 \mathrm{GHz}$ at E plane

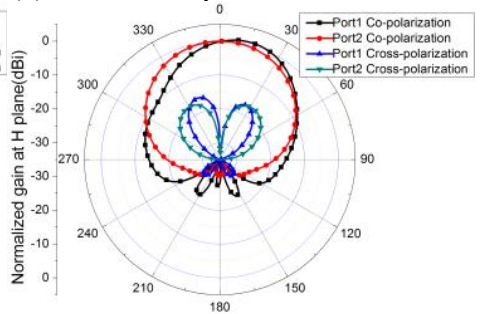

(f) $12 \mathrm{GHz}$ at $\mathrm{H}$ plane

Figure.10 Radiation patterns of the proposed antenna

\section{Summary}

The planar slot antenna has an advantage of low profile and by choosing the appropriate slot form it can achieve a quite broad band. This paper designs a round slot antenna and introduces differential feed to suppress the cross polarization level, which achieves a relatively broad pattern band. On the basis, the paper proposes a multi-layer slot structure to improve the port isolation under the condition of co-planar dual-polarization. The slot antenna achieves a $25.4 \%$ common impedance bandwith, and the port isolation is below $-20 \mathrm{~dB}$ within the working band. The patterns have a good consistency and the XPL is below $-15 \mathrm{~dB}$ from $9.3 \mathrm{GHz}$ to $12 \mathrm{GHz}$. 


\section{References}

[1] Kraus J, Marhefka R. Antennas for All Applications, 3/e[J] , 2006.

[2] Hirokawa J, Ando M, Goto N. Waveguide-fed parallel plate slot array antenna[J]. IEEE Transactions on Antennas \& Propagation, 1992, 40(2):218-223.

[3] Suh Y H, Park I. A broadband eccentric annular slot antenna[C]// Antennas and Propagation Society International Symposium. 2001:94-97 vol.1.

[4] Mobashsher A T, Islam M T, Misran N. Loaded Annular Ring Slot Microstrip Antenna For Wideband And Multi-band Operation[J]. Microwave Journal, 2011, 54(9):146-158.

[5] Tiang S S, Ain M F, Abdullah M Z. Microstrip-fed low profile and wideband wide-slot antenna for breast imaging[C]// Microwave Conference Proceedings. 2012:926-928.

[6] Li W, Chen X J, Zhang B, et al. Dual-polarised cavity-backed annular slot antenna of compact structure[J]. Electronics Letters, 2014, 50(23):1655-1656.

[7] Zhang Q, Jiang D, Wu W. An Integrated Diversity Antenna Based on Dual-Feed Cavity-Backed Slot[J]. IEEE Antennas \& Wireless Propagation Letters, 2014, 13(1):301-304.

[8] Lian R, Wang Z, Yin Y, et al. Design of a Low-profile Dual-Polarized Stepped Slot Antenna Array for Base Station[J]. IEEE Antennas \& Wireless Propagation Letters, 2015, 15:1-1. 